\title{
Tensiones socioambientales y expansión de la frontera extractiva: el caso de la política de protección a los PIACI en el Proyecto Camisea
}

\section{Socio-environmental tensions and expansion of the extractive frontier: the case of the policy of protection of the PIACI in the Camisea Project}

\author{
Alejandro Mejía Tarazona ${ }^{1}$ \\ FLACSO - Ecuador
}

\section{Resumen}

El avance del extractivismo en América Latina luego del boom de los commodities ha puesto sobre la agenda pública la problemática del aumento de los conflictos socioambientales en la Amazonía, en un escenario complejo de renovadas disputas entre Estado, empresas y pueblos indígenas. El

1 Magíster en Políticas Públicas (FLACSO - Ecuador). Profesor en la Facultad de Derecho y Ciencia Política de la Universidad Nacional Mayor de San Marcos (UNMSM) y miembro del Grupo de Investigación en Políticas Públicas Comparadas (FLACSO - Ecuador).

Correo: ramejiafl@flacso.edu.ec. 
presente artículo ${ }^{2}$ aborda la discusión entre política pública y responsabilidad estatal para el caso de los pueblos indígenas en aislamiento y contacto inicial en el Proyecto Camisea. El argumento principal es que, si bien el Estado peruano ha innovado en el diseño de políticas para la protección de los PIACI, la existencia de un marco institucional difuso ha permitido el surgimiento de conflictos socioambientales debido a la ampliación de las fronteras extractivas y afectaciones de derechos fundamentales, sociales y colectivos. Este trabajo se posiciona en un contexto de incremento de tensiones socioambientales producto de los avances de las actividades extractivas en Camisea.

Palabras clave: Gobernanza ambiental, pueblos indígenas, Amazonía, gas, derechos colectivos

\section{Abstract}

The advance of extractivism in Latin America after the boom of commodities has put on the public agenda the problem of the increase of socio-environmental conflicts in the Amazon, in a complex context of renewed disputes between the State, companies and indigenous peoples. This article addresses the discussion between public policy and state responsibility for the case of indigenous peoples in isolation and initial contact in the Camisea Project. The main argument is that, although the Peruvian State has innovated in the design of policies for the protection of the

2 Agradezco a Kelly Torres, estudiante de la Escuela de Ciencia Política en la UNMSM, por su ayuda en la recopilación de información y bibliografía para esta investigación. 
PIACIs, the existence of a diffuse institutional framework has allowed the emergence of socio-environmental conflicts due to the expansion of extractive borders and effects of fundamental, social and collective rights. This work is positioned in a context of increased socio-environmental tensions resulting from the increase of extractive activities in Camisea.

Key words: Environmental governance, indigenous peoples, Amazon, gas, collective rights.

\section{Introducción}

La profundización del extractivismo en la Amazonía y las nuevas formas de gobernanza de los recursos naturales, ha traído consigo un mayor interés en las agendas de investigación por el estudio de los pueblos indígenas y sus dinámicas frente al Estado y las empresas transnacionales. Sin embargo, aún existe poca investigación con respecto a los pueblos indígenas en aislamiento voluntario y contacto inicial, categoría señalada para aquellas etnias o grupos tribales que han optado por el principio de no contacto como derecho a su libre determinación. Esto abre una discusión sobre la responsabilidad estatal y la eficacia de los marcos institucionales para la protección y reconocimiento de derechos fundamentales y colectivos para los PIACI, ${ }^{3}$ frente al avance de la frontera extractiva en la región amazónica.

3 Pueblos Indígenas en Aislamiento y Contacto Inicial. 
En un contexto donde el modelo neoextractivista se ha consolidado como la base del desarrollo de las democracias latinoamericanas (Lander, 2014), este fenómeno ha dado origen a un aumento considerable de los conflictos socioambientales, renovando las disputas territoriales, resistencias sociales y afectaciones a los derechos sociales y colectivos de los pueblos indígenas (Merchand, 2016). Frente a la reprimarización de la economía y el aumento de las actividades extractivas, los gobiernos han tenido avances limitados con respecto a precautelar derechos fundamentales y colectivos de los pueblos indígenas, lo que se ha traducido en el aumento de tensiones ambientales, criminalización de las protestas y movilización de organizaciones indígenas (Paredes y de la Puente, 2014; Orihuela, 2014). Así, en el Perú se ha comenzado a plantear una agenda de investigación que permite entender la relación entre sociedad y naturaleza, para consolidar los estudios ambientales dentro de las Ciencias Sociales (Damonte y Vilas, 2014).

El presente artículo parte de la interrogante de por qué la responsabilidad estatal explica el aumento de los conflictos socioambientales en la Amazonía. Para ello, se plantea que, si bien el Estado peruano ha innovado en el diseño de políticas para la protección y reconocimiento de los PIACI, la existencia de un marco normativo difuso y el interés del

56 Estado por expandir la frontera extractiva, ha permitido la emergencia de conflictos y tensiones socioambientales. La metodología se basa en el análisis de los instrumentos de política para. La metodología se basa en un análisis descriptivo en base a los instrumentos de política como evidencias empíricas. El caso de estudio es la política pública para los pueblos indígenas en aislamiento y contacto inicial (PIACI) en el Perú. 
Para ello, se esboza la siguiente estructura. Primero, una discusión teórica desde los conflictos socioambientales y la política pública para abordar la problemática planteada en líneas anteriores. Seguidamente, se explica la trascendencia del Proyecto Camisea para el sector hidrocarburífero en el Perú y la aparición de los PIACI como nuevo actor en la gobernanza de los recursos naturales. En tercer lugar, se aborda el rol del Estado en el diseño de políticas para los PIACI y el surgimiento de tensiones socioambientales en el Consorcio Camisea. Finalmente, se plantean algunas conclusiones a partir del caso estudiado.

\section{Conflictos socioambientales y política pública: una aproximación teórica}

En la última década, el aumento de las actividades extractivas en la Amazonía ha puesto sobre la agenda pública la problemática de la respuesta estatal frente al incremento de los conflictos socioambientales, teniendo a los pueblos indígenas como los principales afectados por el avance de la frontera extractiva. Esto abre la discusión sobre la responsabilidad estatal, mediante el diseño de políticas públicas, y su relación con el aprendizaje institucional con respecto al manejo de los conflictos socioambientales. En este sentido, la idea que se tiene sobre la institucionalización de políticas de protección hacia los PIACI desde los distintos actores involucrados, va a generar tensiones en lo que respecta a la gobernanza, lo que deviene en el surgimiento de tensiones ambientales durante los últimos años.

Aproximarse a las dimensiones e interacciones que caracterizan a un conflicto socioambiental, se hace necesaria, en pri- 
mer lugar, para una compresión de por qué el conflicto es de un interés permanente en las agendas de investigación en las Ciencias Sociales. En su momento, Dahrenford (1992) planteó una propuesta innovadora desde la sociología para proponer una teoría del conflicto partiendo de entender por qué las sociedades no logran integrarse como tales y como estos conflictos que surgen se deben también a problemas estructurales de las sociedades modernas, así como plantea la necesidad de caracterizar las diversas formas de conflictos existentes. Sin embargo, Coser (1970) estructura nuevos aportes a los planteamientos teóricos existentes sobre el conflicto social, dejando atrás la dicotomía entre consenso y conflicto y comprender que ambos términos están entrelazados y explican el orden político existente.

El conflicto social si bien también trae oportunidades de cambio en un determinado contexto, así como es un conjunto de resultados de disputas históricas, Silva (2008) sostiene la necesidad de construir conceptos propios dentro de la teoría sociológica para abordar las motivaciones e intereses que llevan a la configuración de un conflicto social. En ese sentido, se hace indispensable indagar dentro de los mismos conflictos, identificar a los grupos en disputas, los valores e interés que subyacen al mismo, así como estos pueden promocionar el cambio social en distintos niveles de gobernanza. Esto 58 sugiere que la tipificación de un conflicto por determinadas características es una metodología necesaria para entender las condiciones por la cual surgen los mismos.

En ese sentido, con una tipificación de los conflictos, comenzaron a surgir diversos enfoques en las últimas décadas, siendo una de estas la perspectiva donde lo ambiental comienza a interrelacionarse con lo social. Orellana (1999) señala que los 
conflictos son precisamente procesos ambientales y sociales, ya que hay una afectación de dinámicas políticas, sociales y económicas de los distintos actores inmersos en un conflicto. Se reafirma entonces, que la inclusión del aspecto ambiental en un conflicto, genera un cambio en la interpretación de las relaciones sociales existentes, cuando comienzan a surgir tensiones por el acceso a los recursos.

Frente a este enfoque, se propone una diferenciación entre los conflictos ambientales y conflictos socioambientales. Por un lado, un conflicto ambiental surgiría debido a las consecuencias producidas por el uso del suelo en una actividad extractiva, así como una distribución desigual de las "externalidades". Sin embargo, un conflicto socioambiental tendría en las disputas por el control y acceso a los recursos ambientales (petróleo, gas, minerales) el origen de los mismos (Sabatini, 1996), siendo la diferencia sustancial que, en este tipo de conflicto, las comunidades son directamente afectadas por los impactos y pasivos ambientales de un determinado proyecto extractivo.

Está contraposición de definiciones sobre los tipos de conflictos, no hace más que poner en evidencia que un conflicto ambiental también tiene un componente social, basado en el impacto que este genera en los actores sociales y las minorías étnicas (Fontaine, 2004). Por ello, los conflictos socioambientales van a ahondar en este aspecto, siendo micro espacios donde van a estar contrapuestas una serie de racionalidades y lógicas con respecto a cosmovisiones ambientales y culturales de cada uno de los actores involucrados. Frente a este grado de complejidad cercano a la ética ambiental, el rol de la responsabilidad estatal, a través de políticas públicas para incidir en el manejo y resolución de conflictos socioam- 
bientales, es un factor necesario para mejorar la calidad de la gobernanza.

Las políticas públicas son un factor clave en la capacidad y responsabilidad estatal para atender los problemas que surgen en el espacio público. Hay un consenso general que, con la implementación de las reformas neoliberales en los 90's, el nuevo rol del Estado redujo notablemente su capacidad de implementar políticas públicas exitosas por parte de los gobiernos que, frente al surgimiento de actores no estatales, pone en relieve el problema del déficit democrático (Fontaine, 2015). Así, Estados con una capacidad débil para disenar e implementar políticas públicas y marcos normativos difusos, hacen posible que el incremento de los conflictos socioambientales se configure como un problema público.

Definir que es una política pública es aún una discusión actual en el campo académico. Aguilar (2010) precisa que una política pública es la clase de acción directiva de un gobierno que logra cubrir todos los campos de la responsabilidad estatal, dando mayor trascendencia al aspecto social. También se puede entender como el diseño de una serie de planes de acción orientado al logro de objetivos concretos asumidos por los gobiernos de turno. En ese sentido, una política pública sería una respuesta a demandas de la sociedad, ya sean respecto a derechos o necesidades (Fontaine 2015, 23).

Así, el diseño de políticas públicas no solo dependería de la capacidad estatal, sino también de la dimensión de la responsabilidad estatal, que para cumplir con una serie de objetivos necesita de instrumentos de política eficientes que respondan a las demandas públicas. Es así que, la selección de instrumentos de política de acuerdo a las preferencias de los go- 
biernos, permitiría mejorar los estilos de implementación de las políticas públicas (Hood: 1986; Peters: 2015; Fontaine: 2015). En efecto, el desafío de la toma de decisiones en la acción pública a través de la ejecución de políticas públicas eficientes, tiene en los instrumentos de políticas los medios para responder a los problemas de la gobernanza en los conflictos socioambientales, así como a precautelar derechos de actores no estatales que han cobrado mayor preponderancia en la acción pública.

Según lo propuesto, la interrelación entre políticas públicas y el surgimiento de conflictos tiene como factor causal la capacidad estatal para incidir en un determinado problema público a través del diseño de políticas sustentadas en un marco normativo eficiente. Las demandas insatisfechas de actores fuera de la institucionalidad, se configuran en problemas públicos, que ante un Estado que no cuenta con los instrumentos normativos necesarios para enfrentar escenarios en constante cambio, trae consigo un incremento de las tensiones socioambientales, lo que deviene en una vulneración directa a derechos fundamentales y colectivos. De esta manera, son las políticas públicas las que permiten mejorar la calidad de la gobernanza en un contexto social cambiante, posicionándose como acciones dentro del marco institucional para el manejo de los conflictos socioambientales.

\section{El Proyecto Camisea: extractivismo y nuevas disputas en} la Amazonía

La extracción de recursos naturales ha sido la base del crecimiento económico en distintas etapas de la historia republicana. Los gobiernos de turno encontraron en la profundiza- 
ción de las actividades extractivas la razón fundamental para legitimar los planes para lograr el salto al desarrollo, configurando una sociedad rentista cada vez más dependiente de los precios internacionales ante la demanda de materias primas. Sin embargo, desde el descubrimiento del guano hasta el avance del Estado hacia la Amazonía en busca de recursos naturales, el Estado peruano no había diseñado políticas ante la aparición de un nuevo actor en la gobernanza de los recursos naturales: los pueblos indígenas en aislamiento y contacto inicial. Con el descubrimiento de las Reservas de Camisea a finales de los 80's, las interrelaciones entre Estado y sociedad en la Amazonía cambiarían sustancialmente.

El descubrimiento de las reservas de gas en la Amazonía tiene una relación directa con el interés del Estado peruano por modernizar y llevar el desarrollo hacía regiones donde la presencia estatal era limitada, pero que se configuraba como una nueva fuente de recursos naturales. Esto, más que sentar las bases de un desarrollo sustentable, devino en un proceso prologando de afectación a los derechos de los pueblos indígenas, lo que trajo consigo un aumento de las tensiones y el surgimiento de actores no estatales (organizaciones indígenas) como interlocutores de estos nuevos actores.

Antes que el gas se posicionara como un recurso estratégico 62 para el Estado peruano, el petróleo ocupo un rol imprescindible como parte del desarrollo estatal en gran parte del Siglo XX. La extracción petrolera en el Perú tiene sus orígenes en la primera perforación del pozo de petróleo hallado en Zorritos a finales del siglo XIX, lo cual posicionó al Perú como el primer país en América Latina en sumarse a la extracción de petróleo (Castagnino, 2015). Sin embargo, la ausencia de tecnología, personal calificado y las limitaciones económicas 
del erario público, no generaron la confianza suficiente en la clase política para crear los medios para incentivar la extracción petrolera.

La extracción petrolera tuvo el despegue ansiado con la presencia de capitales transnacionales interesados en las reservas halladas en el zócalo continental a finales de la década de los 20's. Esto significó una etapa crucial para el sector energético en el Perú: la intervención directa del Estado en la explotación y exploración petrolera, así como en el diseño de los primeros marcos institucionales que regularían la extracción del petróleo en el Perú (Candela, 2008). A pesar del interés estatal en liderar las actividades extractivas en el sector, esto devino en un proceso de políticas difusas y la limitada capacidad estatal para responder a la demanda interna, trajo consigo un desarrollo incipiente de la explotación petrolera.

Los fracasos en el hallazgo de nuevas reservas petroleras por parte del Estado y las empresas transnacionales, problemas contractuales entre el Estado peruano y la IPC (International Petroleum Company), así como la crisis de los gobiernos democráticos a mediados de los 60's, tuvo como resultado un retorno hacia políticas nacionalistas bajo la dictadura militar. Así, la economía nuevamente tendría al Estado como su principal protagonista y una de las primeras medidas del gobierno militar fue la de reorganizar las relaciones contractuales en el sector petrolero y consolidar un rol dominante en la gobernanza del sector. Con esto, se daría inicio a una nueva etapa en el desarrollo energético en el Perú: la apertura de la Amazonía a la extracción petrolera (Torres, 2008; Wisse, 2014).

Con la presencia estatal en la Amazonía, comenzarían los primeros contactos con un nuevo actor que reconfiguraría las 
relaciones de gobernanza en la región: los pueblos indígenas en aislamiento y contacto inicial. El avance de las actividades extractiva bajo el liderazgo del Estado y empresas transnacionales, sin un marco institucional que respondiera al contexto bajo el cual se profundizaba la actividad petrolera, generó una serie de impactos en los territorios que se constituían hasta ese momento como espacio vital de los pueblos indígenas originarios. Las dinámicas culturales, sociales y económicas se vieron alteradas por el ingreso del Estado con una idea de desarrollo basado en la extracción de recursos no renovables sin una comprensión de las interacciones existentes entre los pueblos indígenas y la naturaleza.

Bajo ese contexto, el hallazgo de reservas de gas en la Amazonía, determinó que Camisea se convirtiera en el nuevo motor del desarrollo energético para el Estado peruano. Hacia finales de los 80's, un contacto no voluntario entre trabajadores de la empresa Shell y pueblos indígenas que habitaban en el Valle de Urubamba, permitió el descubrimiento de las reservas conocidas hasta hoy en el Proyecto Camisea. No obstante, este contacto no previsto con pueblos indígenas en aislamiento que, al no haber tenido ningún contacto previo con la civilización, trajo como consecuencia que gran parte del pueblo Nahua fuera diezmado a causa de las enfermedades (La Torre, 1998).

64 El Proyecto Camisea y gran parte de los lotes que lo conforman se encuentran ubicados en el Valle de Urubamba, catalogada como una zona de alta sensibilidad biológica y cultural (Ross, 2008). Debido a la presencia de una riqueza en biodiversidad y presencia de pueblos indígenas vulnerables a nuevos contactos, el Estado peruano ve la necesidad de crear un territorio especial bajo la cual precautelar sus derechos al libre desplazamiento y conservación de sus tradiciones y cos- 
tumbres. A inicios de los 90's, en una acción multisectorial realizada para garantizar su espacio de vida, se crea la Reserva del Estado a favor de los grupos étnicos Kugapakori, Nahua y Nanti (Resolución Ministerial Nº046-90), siendo la única reserva con una normativa especial de alcance nacional (Zambrano, 2015)

La llegada de Fujimori al poder a inicio de los 90's, tuvo como uno de sus primeros objetivos una reorganización estructural del sector hidrocarburos, con el fin de dinamizar el sector con el ingreso del capital extranjero, luego del fracaso de las políticas nacionalistas desde los 70's. La liberalización de la economía tuvo en el sector energético a su principal protagonista, guiado por los lineamientos del gobierno de turno que buscó recobrar la competitividad sectorial, ahora con un rol mínimo del Estado en la gobernanza energética. Un nuevo marco institucional acorde a las necesidades estructurales del sector para insertarse nuevamente en el mercado internacional y con un manejo interno regido por el derecho privado, permitió al Estado peruano recuperar el interés en las reservas gasíferas ubicadas en Camisea.

La regulación sobre el uso de territorios para el desarrollo de la industria extractiva, fue una deuda pendiente del Estado desde su normativa interna. Los esfuerzos de los gobiernos nacionalistas en los 70's no fueron suficientes como posicionar en la agenda pública problemáticas como los derechos fundamentales de los pueblos indígenas en la Amazonía. No será hasta 1993, que bajo un nuevo gobierno que respondía a lineamientos de gestión ambiental auspiciados por los organismos internacionales como el BID y el Banco Mundial, que se establece un sucedo trascendental para la gobernanza de los recursos naturales. Bajo la Resolución Legislativa 
No 26253, el Congreso Constituyente de 1993 aprobó la adopción del Convenio 169 de la OIT sobre pueblos indígenas y tribales. Este instrumento legal sería ratificado en 1994 y no entraría en vigor hasta inicios de 1995, con lo que pasa a ser parte de la normativa interna y bajo un rango constitucional (Aranda, 2012).

Materializados los avances en cuanto a un reconocimiento de los derechos de los pueblos indígenas con la adopción del Convenio 169 a la normativa peruana, la explotación de las reservas de Camisea ya era un tema estratégico en la agenda gubernamental para reactivar el sector hidrocarburos. Esto llevó a que el gobierno de Fujimori suscribiera inicialmente un contrato entre PetroPerú y la empresa Shell para evaluar el potencial gasífero de los yacimientos. Sin embargo, al cabo de dos años, la empresa Shell abandonó el Proyecto, por lo que el liderazgo y activos del Proyecto regresaron a la administración del Estado peruano. Esto llevó a que la COPRI (Comisión de Promoción de la Inversión Privada), en conjunto con la Comisión especial creada para Camisea (CECAM), convocaran a licitaciones públicas internacionales bajo una nueva modalidad segmentada de negocios, siendo PLUSPETROL la empresa ganadora de la misma y a la cual se le concesionaba los derechos de exploración y explotación en los Lote 88 y 56 a partir del ańo 2000 66 (Gamboa et al, 2008).

\section{Tensiones socioambientales y gobernanza ambiental: la protección y reconocimiento de los PIACI en Camisea}

Los conflictos por el control y acceso a los recursos en la Amazonía, así como las disputas territoriales, ha generado 
un proceso de enfrentamiento entre los actores involucrados a partir de distintos intereses e ideas sobre la naturaleza, el territorio y las formas de precautelar derechos colectivos. Mejía (2017) sostiene que en Camisea se ha configurado un conflicto de contenido ambiental debido a vulneraciones al territorio de los PIACI, expansión de la exploración de lotes gasíferos, así como la identificación de PLUSPETROL como el actor causante del impacto ambiental y la alteración de la estabilidad histórica entre los grupos étnicos y su hábitat. Así, con la intensificación del proceso de ampliación de la frontera extractiva, ha determinado que en Camisea emergiera un conflicto socioambiental, la cual constituye un escenario de tensiones ambientales entre instituciones, actores sociales y empresas.

Con la concesión de los derechos de explotación, distribución y transporte a distintas transnacionales a cargo del Proyecto Camisea, el Estado peruano planteo una serie de lineamientos para asegurar el abastecimiento del mercado nacional del gas, siendo esta una medida estratégica para reactivar el sector hidrocarburos como uno de los motores del desarrollo. El avance de la frontera extractiva y una institucionalidad inexistente para precautelar derechos de los pueblos indígenas en aislamiento y contacto inicial fue el contexto bajo el cual se desarrollarían los primeros ańos de explotación de las reservas gasíferas del Lote 56 y 88. El marco institucional en el sector hidrocarburos había tenido avances limitados durante los 90's en torno a la protección de derechos de pueblos indígenas, a pesar de que desde 1996, el Convenio 169 de la OIT ya había sido incorporado al ordenamiento jurídico del Estado peruano.

Los primeros avances con respecto a la institucionalidad para los derechos de los PIACI surgieron en el sector hidrocar- 
buros, concentrándose ello en los requerimientos existentes para la Evaluación de Impacto Ambiental (EIA). De acuerdo a la Ley $N^{\circ}$ 27446, que tiene como finalidad la creación de un Sistema Nacional de Evaluación del Impacto Ambiental (SEIA), siendo una de sus directrices el control de los impactos ambientales negativos por el proyecto extractivo. Precisamente, en el artículo 5, se hace referencia a que uno de los criterios principales del EIA es velar por la protección de sistemas y estilos de las comunidades indígenas, siendo estas obligaciones supervisadas por el Consejo Nacional de Ambiente (CONAM).

Luego de la caída de Fujimori, Alejandro Toledo había llegado al poder con una agenda que permitiría mayor participación de las comunidades indígenas y proyectos para diseñar marcos institucionales para su reconocimiento y protección. La creación del CONDEPA y el INDEPA, no tuvieron el protagonismo esperado para impulsar la agenda de las organizaciones indígenas. Sin embargo, será desde el sector hidrocarburos, que mediante la Resolución Ministerial 596 del Ministerio de Energía y Minas, se crea un dispositivo legal que regulan los procesos de participación y consulta previa para los pueblos indígenas en los proyectos extractivos. Empero, siendo los pueblos indígenas en aislamiento y contacto inicial actores que no poseen el pleno reconocimiento del Es-

68 tado moderno y sus instituciones, genera vacíos en una institucionalidad que los reconoce como pueblos indígenas con una categoría distinta a otros pueblos indígenas reconocidos como actores y representados por organizaciones indígenas como AIDESEP.

En los inicios del gobierno de Toledo se conformó la Comisión Especial Multisectorial paras las Comunidades Nativas, 
la cual diseñó un Plan de Acción la cual estableció como un tema prioritario establecer un régimen jurídico especial para los pueblos indígenas en aislamiento voluntario, siendo dos directrices principales garantizar su protección y seguridad jurídica de sus territorios, que permitieran un proceso paulatino de interacción con la sociedad. Estos objetivos se establecieron a través del Decreto Supremo N 028 - 2003, la cual cambiaría ciertas especificaciones y elevaría el nivel de protección a los pueblos indígenas que habitan en la Reserva del Estado a favor de los grupos étnicos Kugapakori y Nahua, espacio donde se desarrollan las actividades extractivas vinculadas al Consorcio Camisea.

Este dispositivo legal establece nuevas disposiciones jurídicas con respecto al marco normativo diseñado en los 90's para la protección de los PIACI en la Reserva a favor del Estado. Se agrega la categoría de "Reserva Territorial" y se establece la protección a los grupos étnicos en aislamiento y contacto inicial. Asimismo, se disponen medidas difusas con respecto al desarrollo de actividades extractivas dentro de ese territorio. En el artículo 3, se prohíbe el desarrollo de actividades económicas, así como el otorgamiento de derechos para la extracción de recursos naturales, siendo la CONAPA e INRENA las instituciones encargadas de velar el cumplimiento de las normas establecidas. Sin embargo, el espacio en la cual se desarrollan las actividades de extracción en los Lotes 56 y 88, están superpuestas al territorio protegido legalmente por el Estado a favor de los PIACI.

En este contexto en la cual se desarrollaban los esfuerzos limitados desde el Estado para la protección de los PIACI en las zonas próximas a Camisea, en el sector hidrocarburos se consolidaron algunos cambios en su normativa interna con 
respecto a la participación en la gobernanza de los recursos naturales. La Resolución Ministerial 535 del MEM dispuso el fortalecimiento en los mecanismos de participación de pueblos indígenas y comunidades nativas en el sector energía, que comprendían las actividades extractivas vinculadas a petróleo y gas. Asimismo, una medida interna dentro del sub-sector en el 2006, limitó el abastecimiento del mercado interno a un período de 20 años, para las empresas a cargo del Consorcio Camisea.

Hacia finales del gobierno de Toledo, da lugar un hito en la protección de los PIACI en la legislación peruana. La Ley 28736 significó un punto de partida para la protección y reconocimiento de derechos fundamentales, sociales y colectivos de los PIACI, la cual se complementaría con el diseño posterior del Reglamento. El nuevo marco normativo definió una categorización de los pueblos indígenas en la Amazonía, resaltando las diferencias entre "aislamiento, “contacto inicial” y "Reservas Indígenas". El cambio sustancial se da al introducir el concepto de Reservas Indígenas, que tal como lo precisa el artículo 5, son intangibles, se prohíbe la relación de cualquier actividad ajena a las costumbres y usos ancestrales de los pueblos indígenas, así como se niegan los derechos para el aprovechamiento de recursos naturales. Sin embargo, esta última disposición deja abierta

70 la posibilidad de que si se hallara un recurso natural cuya explotación sea de necesidad pública del Estado, se proceda bajo los marcos legales existentes.

Esta normativa, aunque innovadora en su diseño e implementación, no establece un carácter jurídico de intangibilidad completa, abriendo la posibilidad a que, ante nuevos hallazgos de recursos no renovables, se pueda permitir la 
expansión de la frontera extractiva en territorios legalmente protegidos por el Estado peruano. Desde la ética ambiental, esto supondrían un cambio en las dinámicas existentes, ya que bajo estas medidas se contemplaría una pugna entre las racionalidades del Estado y los PIACI, con respecto a la gobernanza ambiental y de los recursos naturales en Camisea (Mejía, 2017). Por ello, este dispositivo legal más que establecer mecanismos institucionales para el reconocimiento y protección de los PIACI, mantuvo limitaciones al delegar su cumplimiento efectivo a órganos estatales con una institucional débil, así como un vacío normativo con respecto a la intangibilidad de los territorios con proyectos extractivos ya asentados en la zona, como el caso del Consorcio Camisea.

Durante el gobierno de Alan García, el interés estatal estuvo marcado por una nueva percepción de la Amazonía como fuente de recursos naturales que pudieran sentar las bases para el despegue económico del Perú en un contexto de incremento de la demanda por materias primas en el mercado internacional. Una institucionalidad débil en materia de pueblos indígenas y los sucesos que devinieron en el Conflicto en Bagua, puso en evidencia la deficiencia en la capacidad estatal por construir espacios dentro de los marcos institucionales para el diálogo y reconocimiento de los pueblos indígenas y sus organizaciones representativas (Dolorier y Paneque, 2013). Bajo este escenario, el hallazgo de nuevas reservas de gas en el Lote 57, daría inicio a la expansión de la frontera extractiva en Camisea, así como a nuevas propuestas para explorar nuevos yacimientos próximos a los Lotes 56 y 88.

En efecto, bajo este contexto, la Corte Interamericana de Derechos Humanos $(\mathrm{CIDH})$ ha señalado por varios años conse- 
cutivos que los Estados tienen que tomar medidas adecuadas cuando se otorgan concesiones para desarrollar actividades extractivas (Eguiguren, 2016). Así, en el 2009, la CIDH recomienda al Estado peruano no otorgar ni aprobar proyecto de inversión alguno que pueda afectar la supervivencia de pueblos indígenas o pueblos indígenas o tribales y sus costumbres ancestrales. Los lineamientos establecidos por la CIDH no fueron implementados por el Estado peruano y ante la necesidad pública de explotación de las reservas gasíferas descubiertas en el 2008, prevalecer las medidas para profundizar las actividades extractivas en Camisea sería el detonante para el surgimiento de tensiones ambientales y sociales.

La creación del Ministerio de Cultura a mediados del 2010, debido a la presión pública y las demandas de mayor atención a los pueblos indígenas a raíz de los sucesos de Bagua en el 2009, permitió visibilizar una política pública enfocada a la protección de los PIACI, a través de disposiciones desde la misma entidad rectora. El diseño organizacional de esta nueva entidad estatal trajo consigo la creación de direcciones institucionales que respondieran a las problemáticas de los PIACI, trabajando en conjunto con Perupetro y el Ministerio de Energía y Minas, entidades sectoriales con mayor experiencia en relaciones con los pueblos indígenas. Pese a los múltiples esfuerzos del MINCUL, el ser una institución joven dentro

72 del aparato estatal y con una asignación presupuestaria limitada $(0.1 \%$ del $\mathrm{PBI})$, pone en discusión la capacidad de incidencia estatal frente al aumento de tensiones socioambientales en los proyectos extractivos en la Amazonía.

La Ley de Consulta Previa (2011) y el diseño del Reglamento (2012) fueron instrumentos legales que permitieron institucionalizar en la normativa interna los compromisos interna- 
cionales asumidos por el Estado peruano con la adopción del Convenio 169 de la OIT, convirtiendo así al Perú en el único país en contar con una Ley de Consulta Previa (Blanco, 2016). Pese a estas innovaciones dentro del marco institucional, la interrelación entre pueblos indígenas, Estado y empresas es aún un problema en la agenda pública, en la cual las formas de protección de derechos y territorio, aún sigue siendo difusa y sin objetivos concretos que permitan mejorar la gobernanza ambiental en los proyectos petroleros y gasíferos.

Desde el Viceministerio de Interculturalidad en el Ministerio de Cultura, se dieron medidas sectoriales para afrontar la problemática de los PIACI durante el gobierno de Humala. La promulgación de la Resolución Ministerial 004 en 2013, permitió complementar las disposiciones de la Ley y Reglamento de los PIACI, precisando la necesidad de crear un Registro de los PIACI y de las Reservas Indígenas, con el fin de mantener actualizados los registros oficiales. Asimismo, en 2014, mediante el Decreto 001, se hace un Reconocimiento de los PIACI, y precisando según el artículo 3, el Reconocimiento de los Pueblos Indígenas en situación de aislamiento o en situación de contacto inicial en la Reserva Territorial Kugapakori, Nahua y Nanti.

Siendo el MINCUL la entidad rectora encargada para la protección de los PIACI, en el 2016 se logra publicar el informe "Los Pueblos Indígenas en Aislamiento y Contacto Inicial en la Amazonía Peruana: mecanismos para la protección de sus derechos", como evidencia de implementación de la normativa emitida en años anteriores. Más allá del reconocimiento, categorización e información etnográfica sobre los PIACI que se explican en dicho documento, se señala una 
agenda pendiente en la gestión de los PIACI, siendo la ausencia de un Plan Nacional para protección de los PIACI, así como la incorporación de la categoría de Reserva Indígena a la normativa estatal, los principales retos en la responsabilidad estatal para dotar de mayor protección jurídica. En ese sentido, con una política sin las competencias y lineamientos adecuados en sus objetivos, el aumento de las tensiones socioambientales en Camisea comienzan a originarse a raíz del licenciamiento para expandir los yacimientos gasíferos.

En el 2015, Aministía Internacional revela en su informe mundial sobre los derechos humanos un aumento de los conflictos sociales y protestas por las comunidades afectadas directamente por proyectos extractivos. Asimismo, se precisa que las inconsistencias y ausencia de una metodología clara para la aplicación de la Ley de Consulta Previa en Perú, ha permitido una afectación directa de derechos fundamentales y colectivos de los pueblos indígenas. Del mismo modo, como ya se había especificado el interés estatal por ampliar la explotación en los yacimientos de Camisea en el 2008, en este informe se evidencia que el otorgamiento de la licencia para ampliar la frontera extactiva en Camisea, ha generado un clima de tensiones e intranquilidad entre los pueblos indígenas por posibles afectaciones a sus derechos y territorio (La República, 2015). ${ }^{4}$

Estos pronunciamientos por parte de organizaciones internacionales establecían que los impactos producto de la expansión de las actividades en Camisea se darían ante la ausencia de un marco normativo con mayor relevancia

4 "Advierten que no respetan los derechos indígenas" https://larepublica. pe/politica/858702-advierten-que-no-respetan-los-derechos-indigenas 
institucional que permita precautelar los derechos de los PIACI. En ese sentido, a inicios de 2016, Survival Internacional precisaba que casi un $80 \%$ de los indígenas nahuas, pueblo indígena que habita en la Reserva Territorial próxima a Camisea, padecían envenanimiento tóxico por mercurio, a causa de la expansión de la explotación de las reservas de gas en Camisea (Kaosenlared, 2016)..$^{5}$ De igual forma, se reportaron afectaciones directas a mujeres en los proyectos extractivos, siendo los problemas de salud y abuso de mujeres por parte de trabajadores foráneos, las situaciones más recurrentes en las aproximaciones de la zona de Camisea en Cuzco (La Prensa, 2016). ${ }^{6}$

Con la creación del distrito de Megantoni mediante la Ley $N^{\circ} 30481$ a finales del gobierno de Humala en 2016, los lotes gasíferos que conforman el Consorcio Camisea pasarían a formar parte de esta nueva circunscripción territorial. Bajo este nuevo contexto de cambios territoriales en las inmediaciones de la Reserva Territorial a favor de los PIACI, a partir de 2018 comenzarían a darse nuevos episodios de tensiones entre los pueblos indígenas y la empresa, que llevaría a medidas de radicalización en sus protestas. Al igual que los sucesos de 2015, se reportaron nuevamente incidentes de contaminación por mercurio afectando ahora a la etnia Nahua, uno de los PIACI que habita en la

5 "Indígenas nahuas mueren envenenados con mercurio en Perú" http:// kaosenlared.net/indigenas-nahuas-mueren-envenenados-con-mercurioen-peru/

6 "Perú: contaminación y violaciones, legado de extractivismo para mujeres de Amazonía" https://laprensa.peru.com/actualidad/noticia-medioambiente-peru-amazonia-mujeres-66940 
Reserva Kugapakori, Nagua y Nanti (RPP Noticias). ${ }^{7}$ Esto se dio debido a dos causantes sustentadas en un informe del MINSA, la cual señala que pudo haber originado por consumo de pescado contaminado o directamente por las actividades en Camisea, principalmente con el diseño de la planta de Malvinas, la cual incluyó la instalación de una torre de remoción de mercurio, que habría instalado la empresa PLUSPETROL a partir del licenciamiento obtenido para expandir la explotación de las reservas gasíferas más allá de los Lotes 88 y 56.

Del mismo modo, la radicalización de las medidas de protesta se llevaron a cabo por un suceso de impacto ambiental, a causa de la rotura de un ducto en Camisea, la cual produjo la fuga de líquidos de gas natural a inicios de $2018 .^{8}$ Esto trajo consigo una serie de negociaciones para lograr un acuerdo entre las comunidades nativas y la empresa por el daño ambiental y el monto correspondiente a indemnizaciones. Así, el impacto producido por la ampliación de los yacimientos de gas en Camisea, no solo estarían generando afectaciones directas a los PIACI, sino también a comunidades indígenas próximas a las zonas de transporte del gas natural, lo que configurarían nuevos conflictos socioambientales a raíz de actividades directamente vinculadas al Consorcio Camisea.

7 "Camisea niega responsabilidad en contaminación por mercurio de etnia indígena"

https://rpp.pe/peru/ucayali/camisea-niega-responsabilidad-en-contaminacion-por-mercurio-de-etnia-indigena-noticia-1101600

8 "TgP y nativos siguen enfrentados por derrame en la selva de Camisea" https://larepublica.pe/sociedad/1198864-tgp-y-nativos-siguen-enfrentados-por-derrame-en-la-selva-de-camisea 


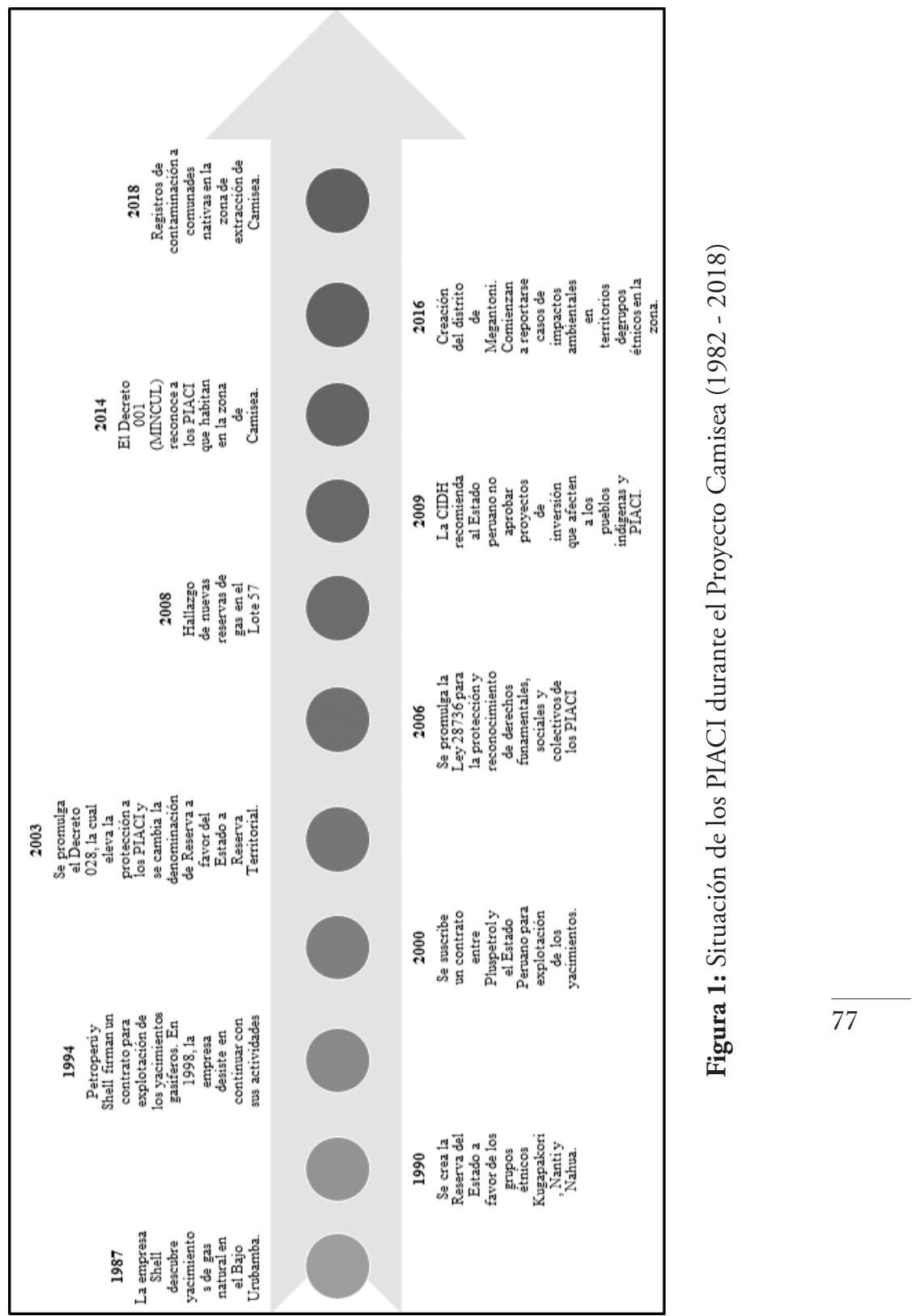




\section{Conclusiones}

De acuerdo al análisis realizado, se logra concluir que existe una relación directa entre la responsabilidad estatal y el aumento de las tensiones socioambientales en el Proyecto Camisea, siendo un favor explicativo el diseño de la política pública, así como la ausencia de planes nacionales estratégicos que permitan materializar los objetivos planteados por la política pública de protección a los PIACI en los marcos normativos. Esto devino del interés estatal por expandir la frontera extractiva en los yacimientos próximos a Camisea, ocasionando alteraciones en el ambiente y territorio, que generan afectaciones directas a los PIACI.

La descripción histórica realizada para entender lo estratégico de Camisea para el sector hidrocarburos y para el desarrollo energético de nuestro país, permite comprender como desde el Estado se entendió durante los 90's el rol de los PIACI como nuevos actores dentro de la gobernanza ambiental. La respuesta estatal fue crear nuevas categorías territoriales las cuales tendrían como objetivo precautelar sus derechos, tradiciones y modos de vida; sin embargo, pese a la adopción del Convenio 169 de la OIT en la normativa interna, no existió un marco institucional adecuado para la protección y reconocimiento de sus derechos fundamentales

78 y colectivos. En definitiva, con la concesión de los yacimientos en Camisea a la empresa Plupestrol a finales de los 90's, comenzaron a surgir los primeros indicios de vulneraciones directas a sus derechos y desplazamiento de los PIACI de sus territorios tradicionales.

Considerando que la política para los PIACI tuvo una institucionalidad débil, no será hasta el retorno a la democra- 
cia que los pueblos indígenas y particularmente los PIACI, serían parte de la agenda pública de los gobiernos de turno. El diseńo de instrumentos innovadores como la Ley de Protección y Reconocimiento de los PIACI, así como su Reglamento, permitió precautelar derechos y territorio, en un contexto de nuevos hallazgos de yacimientos gasíferos en Camisea, así como de un interés estatal por la explotación de recursos naturales en la Amazonía debido a la alta demanda de los mismos en el mercado internacional. Esto trajo consigo nuevos licenciamientos para expandir la exploración y explotación de los nuevos yacimientos, generando un clima de tensiones socioambientales entre los grupos étnicos, representados por organizaciones indígenas, y la empresa Pluspetrol.

A pesar de que desde el Ministerio de Cultura se han dado esfuerzos por implementar los objetivos de la Ley y Reglamento de los PIACI, la ausencia de un Plan Estratégico Nacional y de la inclusión del concepto de Reserva Territorial en la institucionalidad vigente, plantea serios retos para la entidad sectorial encargada de las políticas hacia los pueblos indígenas. Asimismo, los desafíos existen en como estructurar todo el marco normativo frente a los procedimientos y reglamentación que existen para otorgar licenciamiento a nuevos proyectos extractivos en espacios con alta presencia de los PIACI, la cual, según la normativa, prohíbe la concesión de derechos territoriales a empresas transnacionales para la explotación de recursos naturales. Es así que, las evidencias demuestran que la responsabilidad estatal frente a los PIACI no forma parte de una política articulada con otras instituciones sectoriales que son actores estratégicos en la gobernanza ambiental. 
Finalmente, se considera que la emergencia de conflictos socioambientales en Camisea y en la Amazonía, tiene relación con las deficiencias existentes para diseñar marcos institucionales desde el Estado al no tener una concepción clara sobre las dinámicas sociales entre grupos étnicos, instituciones y actores transnacionales. Construir una institucionalidad estatal con la participación de las organizaciones indígenas representativas de los PIACI, va a permitir un mayor conocimiento de las nuevas formas de gobernanza y la interacción entre distintas racionalidades en torno a conceptos como naturaleza, cultura, tradiciones y territorio. La complejidad de los nuevos escenarios que se configuran en la Amazonía, hace necesaria una respuesta estatal que permita fortalecer la justicia ambiental y el acceso a los recursos naturales bajo un marco institucional sostenible. El reconocimiento de la diversidad no solo es un reto para las políticas públicas, sino también para la agenda de investigación con respecto a los estudios socioambientales en el Perú.

Recibido: 10 de agosto del 2018.

Aprobado: 19 de diciembre del 2018.

\section{Bibliografía}

80 Aguilar, Luis (compilador)

(2010) Politica Pública. México DF: Siglo XXI Editores.

Aranda, Mirva

(2012) "Los desafíos de la implementación de la consulta previa en el Perú”. En CAJÍAS DE LA VEGA, Beatriz y HEINS, Claudia (editoras). El Derecho a la Consulta Previa de los Pueblos Indigenas en América Latina. La Paz: Fundación KAS. 
Blanco, Cristina

(2016) "Balance del Perú en el contexto regional: una mirada comparativa del derecho a la consulta previa con relación a Colombia, Chile y Bolivia”. En VARGAS, Karina (compiladora). La implementación del derecho a la consulta previa en el Perú: aportes para el análisis y la garantía de los derechos colectivos de los pueblos indigenas. Lima: GIZ.

Candela, Emilio

(2008) "Los hidrocarburos en el Perú (1931 - 1968)". En Historia del petróleo en el Perú. Lima: Ediciones Copé.

Castagnino, Renzo

(2015) "Formas Contractuales en la Industria de Hidrocarburos Peruana”. Derecho \& Sociedad, Número 35, pp. 84-90. Consulta: 20 de Julio de 2018. http:// revistas.pucp.edu.pe/index.php/derechoysociedad/ article/view/13263/13880

Coser, Lewis

(1970) Nuevos aportes a la teoría del conflicto social. Buenos Aires: Amorrortu.

Dahrendorf, Ralf

(1992) "Hacia una teoría del conflicto social". En ETZIONI, Amitai y ETZIONI, Eva (compiladoras). Los cambios sociales: fuentes, tipos y consecuencias. México DF: Fondo de Cultura Económica.

Damonte, Gerardo, Giselle Vila (Eds.)

(2014) Agenda de investigación en temas socioambientales en el Perú: Una aproximación desde las ciencias sociales. Lima: Centro de investigaciones sociológicas, económicas, políticas y antropológicas (CISEPA)

Dolorier, Gabriela y Pilar, Paneque

(2013) "Poder, gobierno y territorio: análisis del Conflicto de Bagua, Perú". Revista Letras Verdes, Revista Latinoamericana de Estudios Socioambientales, Número 14, pp, 139 - 159. Consulta: 20 de julio de 2018. 
http://revistas.flacsoandes.edu.ec/letrasverdes/article/view/997

Eguiguren, F.

(2016) "Derecho de los pueblos indígenas a la consulta previa: desarrollo, dificultades y desafíos". Pensamiento Constitucional, Volumen 21, Número 21. pp, 61 80. Consulta: 20 de julio de 2018. http://revistas. pucp.edu.pe/index.php/pensamientoconstitucional/ article/view/18700/18941

Fontaine, Guillaume

(2004) "Enfoques conceptuales y metodológicos para una sociología de los conflictos ambientales”. En CÁRDENAS, Martha y Manuel RODRÍGUEZ (editores) Guerra, sociedad y medio ambiente. Bogotá: Foro Nacional Ambiental.

Fontaine, Guillaume

(2015) El análisis de politicas públicas: conceptos, teorias y métodos. Quito: Anthropos, FLACSO Ecuador.

Gamboa, César y Vanessa, Cueto y Jimpson, Dávila (2008) ¿El Estado Peruano cumplió con Camisea? Diagnóstico final sobre el cumplimiento de los Compromisos del Perú en el Ámbito del Proyecto Camisea. Lima: DAR.

Hood, Christopher

(1986) The Tools of Government. Chatham: Chatham House.

Kaosenlared

(2016)

"Indígenas nahuas mueren envenenados con mercurio en Perú". Kaosenlared. Cataluña, 20 de agosto.

Lander, Edgardo

(2014).

El Neoextractivismo como modelo de desarrollo en América Latina y sus contradicciones. Berlín: Heinrich Boll Stiftung.

La Prensa

"Perú, contaminación y violaciones, legado de extractivismo para mujeres de Amazonía". La Prensa. Lima, 02 de diciembre. 
La Republica

(2015) "Advierten que no respetan los derechos indígenas". La República. Lima, 25 de febrero.

La Republica

(2018) "TgP y nativos siguen enfrentados por derrame en la selva de Camisea". La República. Lima, 18 de febrero.

La Torre, Lily

Solo queremos vivir en paz: experiencias petroleras en territorios indigenas de la Amazonía peruana. Lima: IWGIA - Racimos de Ungurahui, Copenhague.

Mejia, Alejandro

(2017) "Los pueblos indígenas en aislamiento y contacto inicial en Camisea (Perú) desde la ética ambiental". En VALLEJO, Ivette y Ramiro ÁVILA (compiladores). El último grito del jaguar: Memorias del I Congreso sobre pueblos indigenas aislados en la Amazonía ecuatoriana. Quito: Ediciones Abya - Yala.

Merchand, Marco Antonio

(2016)

"Neoextractivismo y conflictos ambientales en América Latina”. Espiral, Estudios sobre Estado y Sociedad. Número 66. pp, 155 - 192. Consultado: 20 de Julio de 2018. http://www.scielo.org.mx/pdf/espiral/ v23n66/1665-0565-espiral-23-66-00155.pdf

Orellana, René

(1999) "Conflictos ¿sociales, ambientales, socioambientales?. Conflictos y controversias en la definición de conceptos". En ORTIZ, Pablo (editor). Comunidades y conflictos socioambientales: experiencias y desafíos en América Latina. Quito: Abya Yala.

Orihuela, Juan Carlos

(2014)

"La gobernanza de los recursos y el ambiente en la Amazonía peruana: Delineando una agenda de investigación desde el institucionalismo económico". En DAMONTE, Gerardo y Giselle VILAS (editores). Agenda de investigación en temas socioambientales en 
el Perú: Una aproximación desde las ciencias sociales. Lima: CISEPA.

Paredes, Maritza, y Lorena, De la Puente

(2014) "Protestas y negociaciones socioambientales. El caso de las industrias extractivas". En DAMONTE, Gerardo y Giselle VILAS (editores). Agenda de investigación en temas socioambientales en el Perú: Una aproximación desde las ciencias sociales. Lima: CISEPA.

Peters, Guy (2015)

Advanced introduction to public policy. Cheltenham: Elga.

Radio Programas del Perú

(2018)

"Camisea niega responsabilidad en contaminación por mercurio de etnia indígena”. RPP Noticias. Lima, 25 de enero.

Ross, Catherine

(2008) "El caso del Proyecto Camisea: logros y limitaciones de una coalición ciudadana naciente". En SCURRAH, Martín (editor). Defendiendo Derechos y Promoviendo Cambios. El Estado, las Empresas Extractivas y las Comunidades Locales en el Perú. Lima: Instituto de Estudios Peruanos (IEP).

Sabatini, Francisco

(1996) Conflictos por externalidades y conflictos por el control de los recursos ambientales. Santiago de Chile: Mimeo.

84. Silva, Germán

(2008) La teoría del conflicto. Un marco teórico necesario. Prolegómenos. Derechos y valores. Número 22. pp, 29 - 43. Consultado: 20 de Julio de 2018. http://www.redalyc.org/pdf/876/87602203.pdf

Torres, Víctor

(2008) "El Gobierno Revolucionario de la Fuerza Armada”. En Historia del petróleo en el Perú. Lima: Ediciones Copé. 
Walter, Mariana

(2009) "Conflictos ambientales, socioambientales, ecológico distributivos, de contenido ambiental. Reflexionando sobre enfoques y definiciones". Boletín ECOS, Número 6. pp. 1 - 9. Madrid: CIP-Ecososcial.

Wisse, Carol

(2014) "De la apatía a la vigilancia: la política del desarrollo energético en el Perú". Apuntes: Revista de Ciencias Sociales, Número 56/57. pp, 5-41. http://revistas. up.edu.pe/index.php/apuntes/article/view/547/549

Zambrano, Gustavo

(2015) "Más petróleo, ¿̇más problemas? Crecimiento extractivista en el sector Hidrocarburos en el Perú". Lima, Perú. La Revista. Boletín, Sociedad suiza de americanistas. Número 16. pp, 41-56. 
